\title{
CONSUMO ALIMENTAR DE FAMÍLIAS DE PRÉ-ESCOLARES EM SITUAÇÃO DE (IN)SEGURANÇA ALIMENTAR ${ }^{1}$
}

\author{
FOOD CONSUMPTION AMONG FAMILIES OF PRE-SCHOOL \\ CHILDREN IN SITUATION OF FOOD (IN)SECURITY
}

\author{
CONSUMO DE ALIMENTOS DE LAS FAMILIAS DE NIÑOS \\ PREESCOLARES EN SITUACIONES DE (IN)SEGURIDAD ALIMENTARIA
}

\author{
Mariana Cavalcante Martins ${ }^{*}$ \\ Julliana dos Santos Aires ${ }^{* *}$ \\ Katharine BeZerra Dantas ${ }^{* * *}$ \\ Leidiane Minervina Moraes de Sabino ${ }^{* * * *}$ \\ Maria Dalva Santos Alves**** \\ LORENA BARBOSA XIMENES ${ }^{* * * * *}$
}

RESUMO

Objetivo: Descrever o consumo alimentar de famílias de pré-escolares residentes na zona rural e associar aos níveis de segurança e insegurança alimentar e nutricional no qual estão classificados. Material e Método: Estudo descritivo, correlacional desenvolvido com 200 famílias de pré-escolares atendidas em uma Unidade Básica de Saúde da zona rural de um município de Fortaleza-CE. Os dados foram coletados a partir da aplicação da Escala Brasileira de Insegurança Alimentar. Foram seguidas as normas da Resolução 466/2012. Respeitaram-se os aspectos éticos. Resultados: Dos 200 familiares, 12\% estavam em situação de segurança alimentar, enquanto $88 \%$ foram classificadas com insegurança alimentar. Verificou-se associação estatisticamente significante a partir da relação da situação de insegurança alimentar e o consumo de tubérculos/raízes, leite, derivados do leite, fruta/sucos natural, verduras/legumes, carnes e gordura. Conclusão: A partir da constatação da relação entre insegurança com o consumo alimentar dos familiares de pré-escolares é oportuno a implementação de programas de educação nutricional que favoreçam a adoção de hábitos alimentares saudáveis para a população.

Palavras chave: Consumo alimentar, segurança alimentar e nutricional, enfermagem.

\section{ABSTRACT}

Objective: To describe the food consumption among families of pre-school children in situation of food (in) security in a rural area. Method: A descriptive study conducted with 200 families of pre-school children treated

${ }^{1}$ Trabalho extraído de parte da tese de Doutorado apresentada ao Programa de Pós-Graduação em Enfermagem da Universidade Federal do Ceará/UFC, com apoio financeiro do CNPq.

* Enfermeira. Professora da Universidade Federal do Ceará, Fortaleza, CE. Endereço: Rua Alexandre Baraúna, nº 1115, Rodolfo Teófilo, CEP: 60430-160 - Fortaleza - Ceará, Brasil. Email: marianaenfermagem@hotmail.com

${ }^{* *}$ Enfermeira. Mestre em Enfermagem. Universidade Federal do Ceará, Fortaleza, CE, Brasil. Email: juju_juzita@hotmail.com

${ }^{* * *}$ Acadêmica de Enfermagem. Universidade Federal do Ceará, Fortaleza, CE, Brasil. Email: kate_dantas@hotmail.com

${ }^{* * * *}$ Enfermeira. Docente da Faculdade Integrada da Grande Fortaleza. Mestre em Enfermagem. Universidade Federal do Ceará, Fortaleza, CE, Brasil Email: leidinhamoraes@hotmail.com

${ }^{* * * * *}$ Enfermeira. Professora da Universidade Federal do Ceará, Fortaleza, CE, Brasil. Email: profdalvaalves@uol.com.br

${ }^{* * * * * *}$ Enfermeira. Professora da Universidade Federal do Ceará, Fortaleza, CE, Brasil. Email: lbximenes2005@uol.com.br 
at a Basic Health Unit of a rural municipality of Fortaleza, State of Ceará, Brazil. Data were collected applying the Brazilian Food Insecurity Scale. Regulations of Resolution 466/2012 were observed. Results: Of the 200 families, $12 \%$ were in a food security situation, while $88 \%$ were classified as food insecure. A statistically significant association between food insecurity and the consumption of tubers/roots, milk, dairy products, fruit/natural juices, vegetables/salads, meat and fat was found. Conclusion: Based on the observation of the relationship between food insecurity and food consumption among families of pre-school children, we recommend the implementation of food education programs to encourage the adoption of healthy eating habits for the population.

Key words: Food consumption, food and nutrition security, nursing.

\section{RESUMEN}

Objetivo: Describir el consumo de alimentos de familias de niños en edad preescolar residentes en zonas rurales y asociar a los niveles de seguridad e inseguridad alimentaria y nutricional en que están clasificados. Material y método: Estudio descriptivo correlacional realizado con 200 familias de preescolares atendidos en una Unidad Básica de Salud de un municipio rural de Fortaleza-CE. Los datos fueron obtenidos a traves de la aplicación de la Escala Brasileña de Inseguridad Alimentaria. Fueron seguidas las normas de la Resolución 466/2012. Se respetaron los aspectos éticos. Resultados: De las 200 familias, el 12\% se encontraba en la situación de seguridad alimentaria, mientras que el 88\% fueron clasificados como de inseguridad alimentaria. Una asociación estadísticamente significativa entre la relación de la inseguridad alimentaria y el consumo de tubérculos/raíces, leche, productos lácteos, frutas/jugos naturales, verduras/ensaladas, carne y grasa. Conclusión: En base a la observación de la relación entre la inseguridad alimentaria y el consumo de alimentos de las familias de preescolares es oportuna la aplicación de programas de educación nutricional que fomenten la adopción de hábitos saludables de alimentación para la población.

Palabras clave: Consumo de alimentos, seguridad alimentaria y nutricional, enfermería.

Fecha recepción: 23/09/14 Fecha aceptación: 06/11/15

\section{INTRODUÇÃO}

Nas últimas décadas verificou-se um processo de transição nutricional, que tem sido fator fundamental para aumento do número de casos de sobrepeso e obesidade. Modificações dos hábitos alimentares associadas a alterações demográficas, socioeconômicas e epidemiológicas mostram redução progressiva da desnutrição e aumento da obesidade (1-3).

A epidemia da obesidade se dá, na maioria das vezes, por fator nutricional inadequado consequente ao crescimento descontrolado no consumo de alimentos ricos em gordura e com elevado valor calórico, sendo influenciado pelo poder das mídias, pelo avanço da tecnologia dos alimentos industrializados, dentre outros (4). Além disso, o aumento do poder aquisitivo propiciou um maior acesso desses tipos de alimentos, contribuindo para a prevalência de excesso de peso em todos os grupos etário $(5,6)$.

Um inquérito populacional de 144 países, realizado em 2010, mostrou que 43 milhões de crianças pré-escolares estavam com sobrepeso e obesidade, estando 92 milhões em risco de sobrepeso. Os resultados da pesquisa apontaram um aumento na prevalência mundial de obesidade e sobrepeso, passando de 4,2\% em 1990 para 6,7\% em 2010 (7).

A fase pré-escolar, definida como o período de vida entre 3 e 5 anos, tem grande importância no processo de formação dos hábitos alimentares, pois as crianças já são 
capazes de captar informações acerca da alimentação. Durante essa fase, elas apresentam maior facilidade em assimilar conceitos, apreender o autoconhecimento e a conhecer as normas sociais de comportamento, inclusive a alimentar (8). É o período decisivo para a formação do hábito alimentar, tendendo a permanecer o mesmo por toda a vida adulta. Por isso, a importância de estimular o consumo de uma alimentação variada e equilibrada nessa fase da vida (9).

Alguns fatores podem estar relacionados ao consumo alimentar das crianças, como: trabalho materno, pressões familiares, insegurança alimentar e fatores econômicos (10), podendo assim interferir na seleção dos alimentos, refletindo na quantidade e qualidade dos mesmos.

A Segurança Alimentar e Nutricional tem o objetivo de realizar a promoção da saúde por meio de práticas alimentares saudáveis e da garantia de acessibilidade para toda a população. A alimentação deve estar direcionada em uma prática regular, saudável e de quantidade e qualidade adequadas, não comprometendo o bem estar da família nas demais necessidades básicas (3).

De acordo com a Pesquisa Nacional de Amostra de Domicílios (PNAD) realizada em 2004-2009, pelo Instituto Brasileiro de Geografia e Estatística (IBGE), verificou-se que $30,2 \%$ dos domicílios brasileiros estão em situação de insegurança alimentar (IA). Dentro desse grupo os dados foram analisados em suas três categorias, havendo $18,7 \%$ com insegurança alimentar leve, $6,5 \%$ com insegurança alimentar moderada e 5,0\% com insegurança alimentar grave. Ao realizar-se uma comparação com os índices de IA moderada e grave na área urbana e rural, observou-se maior prevalência na área rural, a qual apresentou proporções de 8,6 e 7,0\%, respectivamente. Já nos domicílios da área urbana encontrou-se percentuais de $6,2 \%$ de insegurança alimentar moderada e 4,6\% de insegurança alimentar grave. Dentre as regiões do Brasil, o Nordeste apresentou maior índice de IA, com 46,1\%, sendo 9,3\% de insegurança alimentar grave (11).

Sabe-se que famílias classificadas como em Insegurança Alimentar influenciam diretamente nos tipos de alimentos consumidos pelas crianças, sendo encontrado na região Nordeste altos índices de insegurança alimentar moderada e grave (12). Diante do exposto, percebe-se a necessidade de conhecer os hábitos alimentares das crianças na fase pré-escolar e associar aos níveis de segurança e insegurança alimentar e nutricional no qual estão classificados.

Para tanto, frente às diferentes realidades existentes no ambiente familiar e à importância em fazer um diagnóstico situacional sobre o consumo alimentar correlacionado com a (in)segurança alimentar, favorecerá a um direcionamento das práticas dos profissionais de saúde, com ênfase no enfermeiro, diante do fato de que é na consulta de puericultura que o profissional de enfermagem pode interagir com os familiares da criança, esclarecendo dúvidas sobre hábitos alimentares saudáveis, medidas de prevenção para insegurança alimentar, bem como distúrbios nutricionais, aspectos esses inerentes e fundamentais para um crescimento e desenvolvimento infantil favorável.

Assim o estudo tem como objetivo descrever o consumo alimentar de famílias de pré-escolares residentes na zona rural e associar aos níveis de segurança e insegurança alimentar e nutricional no qual estão classificados.

\section{MATERIAL E MÉTODO}

Trata-se de um estudo descritivo, correlacional com abordagem quantitativa, desenvolvido em uma Unidade Básica de Saúde (UBS) situada em um distrito da zona rural do município de Maranguape, região metropolitana de Fortaleza - Ceará.

A amostra constituiu-se por toda a popu- 
lação de 200 familiares de crianças pré-escolares atendidas na referida UBS. Os critérios de inclusão adotados foram os familiares que fossem cadastrados na UBS selecionada e que residissem no distrito do estudo. E o critério de exclusão correspondeu aos familiares que tivessem limitação cognitiva que impossibilitasse responder/compreender as perguntas do instrumento.

Para coleta dos dados, realizou-se uma entrevista individualizada com os familiares das crianças, na própria UBS, na qual aplicou-se a Escala Brasileira de Insegurança Alimentar (EBIA). Esta foi adaptada e validada para ser utilizada no Brasil e é composta por quinze perguntas, que admitem sim ou não como resposta, e abordam desde a preocupação em dispor regularmente de comida (insegurança leve) até a ausência total da mesma (insegurança grave) (13), nos três meses precedentes à entrevista.

Cada resposta afirmativa da EBIA corresponde a 1 ponto; e zero ponto para resposta "não" ou "não sabe". A somatória desses pontos permite categorizar a família em situação de segurança alimentar (zero pontos), insegurança alimentar leve ( 1 a 5 pontos), moderada ( 6 a 10 pontos) e grave ( 11 a 15 pontos) (14).

A segunda fase desse instrumento refere-se à análise do consumo de treze grupos de alimentos. Essa investigação permite conhecer a situação alimentar, já que identifica o consumo em seus aspectos qualitativos e quantitativos, ou seja, permite que sejam conhecidos os tipos de alimentos consumidos, bem como a sua frequência (15). Dessa forma, o participante confirma ou não a utilização diária de algum alimento pertencente a cada grupo alimentar (tubérculos/raízes; leite; derivados do leite; ovos; fruta/suco natural; verduras/legumes; leguminosas; carnes; embutidos; açúcar; doces; refrigerante/sucos industrializados; gordura), o que possibilita identificar o tipo de alimentação, bem como a presença de alimentos regionais que são consumidos no cotidiano da família.

Os dados foram organizados em gráficos e tabelas, sendo tabulados e processados no Predictive Analytics Software (PASW), versão 18. Para a análise, utilizou-se a estatística descritiva, por meio de frequências absolutas, relativas e o teste linear by linear, sendo considerado significativo o valor de $\mathrm{p}<0,05$.

A pesquisa foi aprovada pelo Comitê de Ética em Pesquisa da Universidade Federal do Ceará sob o parecer no 98/09. Foram respeitadas todas as recomendações e requisitos éticos previstos para pesquisas envolvendo seres humanos, conforme a resolução $n^{\circ}$ 466/2012, bem como os familiares assinaram o Termo de Consentimento Livre e Esclarecido concordando em participar do estudo (16).

\section{RESULTADOS}

Por meio do Gráfico 1 pode-se observar que dos 200 familiares entrevistados, 24 (12\%) apresentaram situação de segurança alimen$\operatorname{tar}(\mathrm{SA})$, enquanto a maioria foi classificada com insegurança alimentar 176 (88\%), sendo $70(35 \%)$ em insegurança alimentar leve, 58 (29\%) em insegurança alimentar moderada e 48 (24\%) em insegurança alimentar grave.

A partir da Tabela 1 pode-se constatar a associação dos alimentos consumidos diariamente pelos familiares com a situação de (in) segurança alimentar, de acordo com a EBIA. Para tanto, verificou-se uma associação estatisticamente significante entre a (in)segurança alimentar e o consumo de tubérculos/ raízes $(p=0,001)$, leite $(p=0,024)$, derivados do leite $(p<0,0001)$, fruta/sucos natural $(p<0,0001)$, verduras/legumes $(p<0,0001)$, carnes $(\mathrm{p}<0,0001)$ e margarina $(\mathrm{p}=0,009)$. 
Gráfico 1. Distribuição das famílias segundo situação de (in)segurança alimentar ( $\mathrm{n}=200)$. Maranguape-CE, 2009.

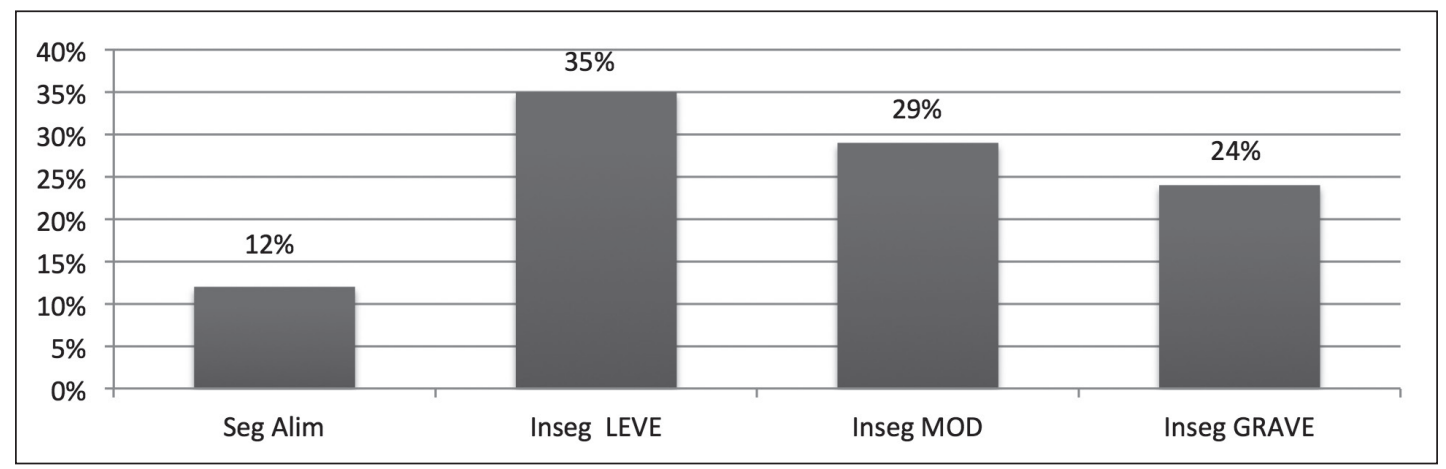

Tabela 1. Associação entre o consumo diário de alimentos pelos familiares e a categorização de segurança alimentar e os três níveis de insegurança alimentar. Maranguape, CE, 2009.

\begin{tabular}{lccccc}
\hline Alimentos & $\begin{array}{c}\text { Segurança } \\
\text { Alimentar }\end{array}$ & $\begin{array}{c}{ }^{\star} \text { IA } \\
\text { Leve }\end{array}$ & $\begin{array}{c}{ }^{*} \text { IA } \\
\text { Moderada }\end{array}$ & $\begin{array}{c}{ }^{*} \text { IA } \\
\text { Grave }\end{array}$ & \multirow{2}{*}{${ }^{* *}$ Valor p } \\
\cline { 2 - 5 } & $\mathrm{N}(\%)$ & $\mathrm{N}(\%)$ & $\mathrm{N}(\%)$ & $\mathrm{N}(\%)$ & \\
\hline Tubérculos/raízes & $14(18,0)$ & $32(41,0)$ & $21(26,9)$ & $11(14,1)$ & 0,001 \\
Leite & $21(12,3)$ & $64(37,4)$ & $50(29,2)$ & $36(21,1)$ & 0,024 \\
Derivados do leite & $18(22,8)$ & $28(35,4)$ & $22(27,8)$ & $11(13,9)$ & $<0,0001$ \\
Ovos & $21(13,8)$ & $55(36,2)$ & $41(27,0)$ & $35(23,0)$ & 0,106 \\
Fruta/suco natural & $23(16,2)$ & $59(41,5)$ & $38(26,8)$ & $22(15,5)$ & $<0,0001$ \\
Verduras/legumes & $22(17,3)$ & $50(39,4)$ & $31(24,4)$ & $24(18,9)$ & $<0,0001$ \\
Leguminosas & $23(12,7)$ & $65(35,9)$ & $51(28,2)$ & $42(23,2)$ & 0,107 \\
Carnes & $23(16,2)$ & $53(37,3)$ & $41(28,9)$ & $25(17,6)$ & $<0,0001$ \\
Embutidos & $16(12,4)$ & $46(35,7)$ & $34(26,4)$ & $33(25,6)$ & 0,941 \\
Açúcar & $23(12,2)$ & $65(34,4)$ & $55(29,1)$ & $46(24,3)$ & 0,948 \\
Doces & $11(16,2)$ & $19(27,9)$ & $23(33,8)$ & $15(22,1)$ & 0,699 \\
Refrig/suco indust. & $18(19,7)$ & $28(30,8)$ & $25(27,5)$ & $20(22,0)$ & 0,068 \\
Gordura & $23(12,7)$ & $67(37,0)$ & $51(28,2)$ & $40(22,1)$ & 0,009 \\
\hline
\end{tabular}

${ }^{*}$ IA: Insegurança Alimentar

** Teste linear by linear

\section{DISCUSSÃO E CONCLUSÃO}

No presente estudo, após classificação realizada por meio da EBIA, verificou-se que apenas $12 \%$ das famílias de crianças pré-es- colares encontravam-se em SA, estando $88 \%$ delas em IA. Por sua vez, na Pesquisa Nacional por Amostra de Domicílios (PNAD), realizada em 2004-2009, identificou-se que $30,2 \%$ dos domicílios brasileiros apresentavam IA. E, em relação às regiões brasileiras, 
o Nordeste obteve um dos maiores índices de IA $(46,1 \%)(11)$.

A diferença percentual entre esses achados torna-se inquietante, pois a prevalência de IA evidenciada na presente pesquisa foi quase o dobro do índice de IA na região Nordeste $(46,1 \%)$ verificado na PNAD. Corroborando com tal fato, pesquisa que comparou a IA em domicílios com crianças menores de sete anos de idade das regiões Sul e Nordeste constatou que a prevalência de IA moderada e grave foi de $7,5 \%$ na primeira; e de $22,9 \%$ na região Nordeste (17).

Diante desta realidade, pode-se inferir que o Brasil ainda apresenta um déficit considerável no que diz respeito à provisão de alimentos e a região Nordeste merece atenção especial, visto que exibe índices elevados de IA. Nesse sentido, a avaliação da prevalência de IA torna-se importante para o planejamento de programas e políticas públicas preventivas, promotoras de saúde e de combate à fome (18).

$\mathrm{Na}$ avaliação da ingestão alimentar das famílias entrevistadas, dos treze grupos de alimentos, os tubérculos/raízes, o leite, os derivados do leite, as frutas/sucos naturais, as verduras/legumes, as carnes e a gordura apresentaram uma tendência de consumo decrescente à medida que IA se agravou, obtendo associação significativa $(\mathrm{p}<0,05)$.

Uma tendência semelhante foi observada em estudo desenvolvido em Duque de Caxias, que analisou a associação entre IA e o consumo alimentar de familiares de crianças de seis a trinta meses de vida, onde constatou-se que aquelas com IA moderada e grave possuíam um baixo consumo de cereais, hortaliças, frutas, carnes e gordura (19).

Ressalta-se que os principais motivos para a ingestão reduzida de cálcio pela população de baixa renda devem-se, provavelmente, ao elevado custo do leite e seus derivados, além de hábitos culturais e alimentares (20).

Pesquisa desenvolvida no Brasil que objetivou descrever a distribuição regional e socioeconômica domiciliar de alimentos no país, evidenciou que a quantidade de calorias correspondeu, em média, a $1.611 \mathrm{kcal} /$ pessoa/dia, sendo $1.973 \mathrm{kcal}$ dos indivíduos no meio rural. Os alimentos básicos de origem vegetal (cereais, leguminosas, tubérculos/raízes) satisfizeram a $45 \%$ das calorias disponíveis para consumo; já os alimentos calóricos (óleos e gorduras vegetais, açúcar, refrigerantes) contribuíram com 28\%; e os produtos de origem animal (carnes, leite e derivados e ovos) com 19\%. Frutas, verduras e legumes contribuíram com apenas 2,8\% das calorias (21), o que caracteriza um consumo insuficiente desse grupo.

Acredita-se, então, que a incorporação de hábitos da sociedade moderna e industrializada no decorrer dos anos, ocasionou um aumento na disponibilidade de alimentos ultraprocessados, tais como biscoitos, refrigerantes e refeições prontas; em detrimento de alimentos minimamente processados, como o arroz, feijão, leite e farinha de mandioca (22).

Com isso, a evolução do padrão alimentar brasileiro vem assinalando um aumento significativo no consumo de alimentos industrializados com alto teor de açúcares, sódio e lipídios (alimentos processados), associado à redução no consumo de alimentos saudáveis.

Mesmo não tendo apresentado, na presente pesquisa, associação estatisticamente significativa com segurança alimentar e o grupo dos embutidos $(p=0,941)$, do açúcar $(p=0,948)$, dos doces $(p=0,699)$ e dos refrigerantes/sucos industrializados $(p=0,068)$, ao comparar o consumo alimentar desses grupos entre o somatório dos três níveis de IA dos familiares observou-se um percentual de consumo de $87,7,87,8,83,8$ e $80,3 \%$, respectivamente. Esse achado assemelha-se ao encontrado em estudo realizado com 177 famílias de São José dos Ramos-PB, o qual identificou que os grupos alimentares citados também não obtiveram associação significativa com a AS (23).

Contudo, é oportuno evidenciar que o acesso da população a alimentos de qualida- 
de e em quantidade suficiente depende, predominantemente, dos preços dos alimentos e da renda familiar (24).

Segundo os resultados da Pesquisa de Orçamentos Familiares (POF), 2008-2009, a renda familiar é um dos fatores determinantes para obtenção de uma alimentação diária adequada. Dessa forma, os indivíduos de classes sociais com renda baixa consomem, em geral, maior quantidade de arroz e feijão. Por outro lado, o consumo do grupo das frutas e verduras aumenta proporcionalmente à renda, assim como o do leite desnatado e dos derivados do leite (25).

Nesse sentido, considera-se a renda mensal familiar como um indicador que proporciona maior impacto na SA. Isso pode ser comprovado em estudo que avaliou a prevalência de IA em área urbana e rural do Brasil e identificou que os domicílios cuja renda per capita correspondia a menos de $25 \%$ do salário mínimo, a condição de IA entre as famílias superava cerca de 3,5 vezes à das famílias com rendimentos de um salário mínimo ou mais (26).

A partir disso, pode-se evidenciar que a análise da distribuição dos gastos e dos preços relativos de categorias e grupos alimentares, mediante parâmetros nutricionais, fornece subsídios à proposição de políticas públicas voltadas para a alimentação saudável (27).

Nesse contexto, o Ministério da Saúde definiu a "promoção da alimentação saudável" como uma das diretrizes da Política Nacional de Alimentação e Nutrição (PNAN) e como eixo estratégico da Política Nacional de Promoção da Saúde (PNPS), e vem criando medidas de incentivo ao consumo de alimentos saudáveis / alimentos regionais (3).

$\mathrm{Na}$ presente pesquisa, entre os grupos de alimentos abordados na EBIA, quatro deles são caracterizados como componentes dos alimentos regionais (frutas; tubérculos/raízes; leguminosas; verduras/legumes). Esses alimentos são típicos de determinada região do país e tem como característica possuir alto valor nutritivo, baixo custo e fácil acesso (28).
Em virtude da carência de alimentos ou por seu consumo inadequado, a população brasileira convive com índices de excesso de peso e distúrbios nutricionais. Dessa forma, enfatizar a valorização do saber popular e da cultura da região estimulando o uso dos alimentos disponíveis torna-se uma das iniciativas que podem contribuir para melhorar o consumo de alimentos saudáveis.

É oportuno evidenciar que a subutilização dos alimentos regionais na alimentação da criança foi constatada em pesquisa (29), a qual destacou, como fator contribuinte, a falta de conhecimento das mães acerca de outras opções de preparo com esses alimentos, ressaltando a necessidade de propagar os benefícios desses alimentos na alimentação da criança e da família.

Nesse sentido, as práticas educativas na perspectiva da promoção da saúde poderão ser uma estratégia importante no âmbito de atuação da enfermagem. Para tanto, são necessários esforços voltados à construção de metodologias inovadoras para os processos educativos, buscando contribuir para a promoção da alimentação saudável, por meio do conhecimento e da problematização da realidade, a fim de desvendar estratégias para adoção de práticas mais saudáveis e adequadas aos contextos de cada grupo social (30).

Em conclusão, a aplicação da EBIA neste estudo permitiu a identificação de prevalência elevada de IA nas famílias do município, sendo esse instrumento uma ferramenta eficaz e de fácil aplicação para população da zona rural.

É essencial a implementação imediata de programas de educação nutricional na tentativa de mudar esse cenário. Além disso, a adoção de estratégias educativas que visem o aumento do consumo dos alimentos regionais poderá contribuir para minimizar os elevados índices de IA no município e para promover hábitos alimentares saudáveis.

Além disso, frente às mudanças nos padrões alimentares das crianças é importante que os profissionais de saúde, dentre eles os 
enfermeiros, busquem promover uma alimentação infantil saudável para a população. Dessa forma, com a orientação alimentar saudável, o profissional poderá envolver a família e a comunidade como sendo coparticipantes na responsabilidade pela alimentação infantil saudável.

Considerou-se como limitação do presente estudo a falta de avaliação do padrão de consumo alimentar em cada refeição diária da família, assim como a não correlação do estado nutricional infantil com os alimentos utilizados, tendo em vista que este poderia constituir em um mecanismo para obter outros resultados para a realização de novas pesquisas e intervenções educativas nesse sentido.

\section{REFERÊNCIAS}

1. Sperandio N, Sant'Ana LFR, Franceschini SCC, Priore SE. Comparação do estado nutricional com utilização de diferentes curvas de crescimento. Rev. Nutr. 2011; 24(4): 565-574.

2. Guimarães NG, Dutra ES, Ito MK, Carvalho KMB. Adesão a um programa de aconselhamento nutricional para adultos com excesso de peso e comorbidades. Rev. Nutr. 2010; 23(3): 323-333.

3. Ministério da Saúde (BR). Política Nacional de Alimentação e Nutrição. Brasília; 2012.

4. Alves LMM, Yagui CM, Rodrigues CS, Mazzo A, Rangel EML, Girão FB. Obesidade infantil ontem e hoje: importância da avaliação antropométrica pelo enfermeiro. Esc. Anna Nery. 2011; 15(2): 238244.

5. Zanini RV, Muniz LC, Schneider BC, Tassitano RM, Feitosa WMN, González-Chica DA. Consumo diário de refrigerantes, doces e frituras em adolescentes do Nordeste brasileiro. Cien Saude Colet. 2013; 18(12): 3739-3750.
6. Levy RB, Castro IRR, Cardoso LO, Tavares LF, Sardinha LMV, Gomes FS et al. Consumo e comportamento alimentar entre adolescentes brasileiros: Pesquisa Nacional de Saúde do Escolar (PeNSE), 2009. Cien Saude Colet. 2010; 15(Supl): 3085-3097.

7. Onis M, Blössner M, Borghi E. Global prevalence and trends of overweight and obesity among preschool children. Am J Clin Nutr. 2010; 92(1): 1-8.

8. Garcia MT, Granado FS, Cardoso MA. Alimentação complementar e estado nutricional de crianças menores de dois anos atendidas no Programa Saúde da Família em Acrelândia, Acre, Amazônia Ocidental Brasileira. Cad Saude Publica. 2011; 27(2): 305-316.

9. Bernart A, Zanardo VPS. Educação nutricional para crianças em escolas públicas de Erechim/RS. Revista Eletrônica de Extensão da URI. 2011; 7(13): 71-79.

10. Lindsay AC, Ferarro M, Franchello A, Barrera RL, Machado MMT, Pfeiffer ME, et al. Child feeding practices and household food insecurity among low-income mothers in Buenos Aires, Argentina. Cien Saude Colet. 2012; 17(3): 661-669.

11. IBGE. Instituto Brasileiro de Geografia e Estatística. Pesquisa Nacional por Amostra de Domicílios: Segurança alimentar (2004-2009). Rio de Janeiro; 2010.

12. Poblacion AP, Marín-Leon L, Segall-Côrrea AM, Silveira JA, Taddei JAAC. Insegurança alimentar em domicílios brasileiros com crianças menores de cinco anos. Cad Saude Publica. 2014; 30(5): 1067-1078.

13. Kepple AW, Segall-Corrêa AM. Conceituando e medindo segurança alimentar e nutricional. Cien Saude Colet. 2011; 16(1): 187-199.

14. Pérez-Escamilla R, Segall-Corrêa AM. Food insecurity measurement and indicators. Rev Nutr. 2008; 21(Supl): 15-26.

15. Melgar-Quinonez HR, Nord M, Perez-Escamilla R, Segall-Correa AM. Psychometric properties of a modified US-house- 
hold food security survey module in Campinas, Brazil. Eur J Clin Nutr. 2008; 62(5): 665-673.

16. Ministério da Saúde (BR). Normas para pesquisa envolvendo seres humanos (Res. CNS no. 466/12) Brasília; 2012.

17. Facchini LA, Nunes BP, Motta JVS, Tomasi E, Silva SM, Thumé E, et al. Insegurança alimentar no Nordeste e Sul do Brasil: magnitude, fatores associados e padrões de renda per capita para redução das iniquidades. Cad Saude Publica. 2014; 30(1): 161-174.

18. Vianna RPT, Segall-Corrêa AM. Insegurança alimentar das famílias residentes em municípios do interior do estado da Paraíba, Brasil. Rev. Nutr. 2008; 21(Supl): 111-122.

19. Antunes MML, Sichieri R, Salles-Costa R. Consumo alimentar de crianças menores de três anos residentes em área de alta prevalência de insegurança alimentar domiciliar. Cad Saude Publica. 2010; 26(8): 1642-1650.

20. Silva CR, Martins BAET, Oliveira VLMI, Miyasaka CK. Consumo alimentar e estado nutricional de Pré-escolares de um centro de educação Infantil do município de São Paulo. Alim. Nutr. 2010; 21(3): 407-413.

21. Levy RB, Claro RM, Mondini L, Sichieri R, Monteiro CA. Distribuição regional e socioeconômica da disponibilidade domiciliar de alimentos no Brasil. Rev Saude Publica. 2012; 46(1): 6-15.

22. Monteiro CA, Levy RB, Claro RM, Castro IRR, Cannon G. A new classification of foods based on the extent and purpose of food processing. Cad Saude Publica. 2010; 26(11): 2039-2049.

23. Silva CCS, Oliveira KBB, Alves AS, Já
JAN, Modesto CAC, Vianna RPT. Associação entre consumo alimentar e (in) segurança alimentar e nutricional em São José dos Ramos - PB. Braz. J. Food Technol. 2012; 15(Supl): 23-30.

24. Nascimento AL, Andrade SLLS. Segurança alimentar e nutricional: pressupostos para uma nova cidadania? Ciênc Cult. 2010; 62(4): 34-8.

25. IBGE. Instituto Brasileiro de Geografia e Estatística. Pesquisa de Orçamentos Familiares 2008-2009: Antropometria e análise do estado nutricional de crianças, adolescentes e adultos no Brasil/IBGE, Coordenação de Trabalho e Rendimento [Internet]. Rio de Janeiro: IBGE; 2010 [citado 10 out 2014]. 127 p. Disponível em: http://biblioteca.ibge.gov.br/bibliotecacatalogo?view=detalhes\&id $=245419$.

26. Mondini L, Rosa TE, Gubert MB, Sato GS, Benício MHD. Insegurança alimentar e fatores sociodemográficos associados nas áreas urbana e rural do Brasil. Informações Econômicas. 2011; 41(2): 52-60.

27. Sarti FM, Claro RM, Bandoni DH. Contribuições de estudos sobre demanda de alimentos à formulação de políticas públicas de nutrição. Cad Saude Publica. 2011; 27(4): 639-47.

28. Ministério da Saúde (BR). Alimentos Regionais Brasileiros. 2a ed. Brasília; 2015.

29. Martins MC, Ximenes LB, Casimiro CF, Silveira VG, Frota MA. Estratégia educativa com enfoque nos hábitos alimentares de crianças: alimentos regionais. Cogitare Enferm. 2009; 14(3): 463-469.

30. Delormier T, Frohlich KL, Potvin L. Food and eating as social practice understanding eating patterns as social phenomena and implications for public health. Sociol Health Illn. 2009; 31(2): 215-228. 